\title{
Pursuing equity in cancer care: implementation, challenges and preliminary findings of a public cancer referral center in rural Rwanda
}

Neo M. Tapela ${ }^{1,3,4,10^{*}}$, Tharcisse Mpunga ${ }^{5}$, Bethany Hedt-Gauthier ${ }^{2,3,4}$, Molly Moore ${ }^{7}$, Egide Mpanumusingo ${ }^{5}$, Mary Jue X $\mathbf{u}^{4}$, Ignace Nzayisenga ${ }^{2}$, Vedaste Hategekimana ${ }^{5}$, Denis Gilbert Umuhizi ${ }^{5}$, Lydia E. Pace ${ }^{4}$, Jean Bosco Bigirimana2, Jing Jing Wang ${ }^{2}$, Caitlin Driscoll ${ }^{8}$, Frank R. Uwizeye ${ }^{2}$, Peter C. Drobac ${ }^{2,3,4}$, Gedeon Ngoga ${ }^{2}$, Cyprien Shyirambere ${ }^{2}$, Clemence Muhayimana ${ }^{5}$, Leslie Lehmann ${ }^{2,3,4,6}$ and Lawrence N. Shulman 2,3,9

\begin{abstract}
Background: Cancer services are inaccessible in many low-income countries, and few published examples describe oncology programs within the public sector. In 2011, the Rwanda Ministry of Health (RMOH) established Butaro Cancer Center of Excellence (BCCOE) to expand cancer services nationally. In hopes of informing cancer care delivery in similar settings, we describe program-level experience implementing BCCOE, patient characteristics, and challenges encountered.

Methods: Butaro Cancer Center of Excellence was founded on diverse partnerships that emphasize capacity building. Services available include pathology-based diagnosis, basic imaging, chemotherapy, surgery, referral for radiotherapy, palliative care and socioeconomic access supports. Retrospective review of electronic medical records (EMR) of patients enrolled between July 1, 2012 and June 30, 2014 was conducted, supplemented by manual review of paper charts and programmatic records.

Results: In the program's first 2 years, 2326 patients presented for cancer-related care. Of these, $70.5 \%$ were female, $4.3 \%$ children, and $74.3 \%$ on public health insurance. In the first year, $66.3 \%(n=1144)$ were diagnosed with cancer. Leading adult diagnoses were breast, cervical, and skin cancer. Among children, nephroblastoma, acute lymphoblastic leukemia, and Hodgkin lymphoma were predominant. As of June 30, 2013, 95 cancer patients had died. Challenges encountered include documentation gaps and staff shortages.

Conclusion: Butaro Cancer Center of Excellence demonstrates that complex cancer care can be delivered in the most resource-constrained settings, accessible to vulnerable patients. Key attributes that have made BCCOE possible are: meaningful North-south partnerships, innovative task- and infrastructure-shifting, RMOH leadership, and an equity-driven agenda. Going forward, we will apply our experiences and lessons learned to further strengthen BCCOE, and employ the developed EMR system as a valuable platform to assess long-term clinical outcomes and improve care.
\end{abstract}

Keywords: Cancer, Implementation, Rwanda, Resource-limited setting, Capacity building, Twinning, Task-shifting

\footnotetext{
*Correspondence: ntapela@gmail.com

'Botswana Ministry of Health, Gaborone, Botswana

${ }^{3}$ Dana-Farber/Brigham \& Women's Cancer Center, Boston, USA

Full list of author information is available at the end of the article
} 


\section{Background}

As cancer-related mortality rapidly outpaces the capacity of developing-world healthcare systems, global health discourse has increasingly encompassed cancer care $[1,2]$. In 2008, cervical cancer and childbirth mortality were comparable [3], and in 2012, the 5.3 million cancer deaths worldwide exceeded those caused by HIV/AIDS, tuberculosis, and malaria combined [1]. Yet, while low- and middle-income countries (LMICs) account for $80 \%$ of disability-adjusted life-years lost to cancer, only $5 \%$ of oncology resources are spent in those countries [2, 4]. Particularly in LMICs, cancer services are inaccessible for most patients, with existing programs located primarily in urban areas or the private sector and focusing on select cancers $[4,5]$. Perhaps with the exception of the AMPATH-Oncology consortium in Kenya [6], models of oncology programs embedded within the public sector and serving rural poor patients are lacking. Furthermore, while general principles in cancer service delivery in resourceconstrained settings have been described $[4,5,7,8]$, few groups outline program-level implementation of oncology services.

Rwanda has greatly improved the health of its 11 million citizens since the catastrophic 1994 genocide [9]. Yet cancer care was extremely limited as recently as 2012, with no oncologist, only one hematopathologist, and three clinical pathologists based in the country. At the time, services were available at only one district hospital and three urban-based national referral hospitals. In 2011 driven by its strong equity agenda and having initiated impressive cervical cancer prevention efforts [10], Rwanda's Ministry of Health ( $\mathrm{RMOH})$ invited Partners In Health (PIH) and Dana-Farber/Brigham and Women's Cancer Center (DFBWCC) to partner in expanding cancer care nationally, targeting poor, rural-based patients. In July 2012, the Butaro Cancer Center of Excellence (BCCOE), a public rural-based facility, was inaugurated by former US President Bill Clinton and the Honorable Minister of Health, Dr. Agnes Binagwaho. Here, we report programlevel description of implementing BCCOE, its preliminary impact and challenges faced in order to share lessons and inform service delivery in similar settings.

\section{Methods: key components to delivering accessible cancer services in a resource-constrained setting} Partnerships

Butaro Cancer Center of Excellence was founded on diverse, long-term partnerships [7, 8]. Spearheading the initiative, the $\mathrm{RMOH}$ set national priorities and coordinated collaborations. $\mathrm{RMOH}$ also provided infrastructure, staff (recruitment of nurses and junior doctors along with salary support for most of them) and non-specialized consumables complementary to oncology services (such as pain medications and intravenous fluids). $\mathrm{PIH}$, an international non-governmental organization with a mandate to deliver healthcare to the most vulnerable communities and extensive experience working in resource-constrained settings [11], was initially invited by $\mathrm{RMOH}$ in 2005 to help expand HIV services to communities in a rural district in the Eastern province. This partnership grew over time to address evolving needs including those in primary care, medical education and specialty-related care such as cancer. In addition to bringing this implementation experience to cancer care, PIH brought on board a network of partners that availed technical expertise in oncology and pathology, funding to support salary for selected staff (such as Rwandan internist and pediatrician), procurement of specialized oncology medications and supplies, pathology equipment and reagents as well as relationships to defray costs (through procurement networks, volunteer clinicians). These partners, including Harvard Medical School, DFBWCC, Jeff Gordon Children's Foundation, The Breast Cancer Research Foundation, LIVESTRONG, and GlaxoSmithKline, are diverse in scope and committed to long term partnerships.

\section{Setting and infrastructure}

Butaro Cancer Center of Excellence is housed within Butaro hospital, a rural district hospital in Burera district (which is home to 321,000 people) located in northern Rwanda approximately $93 \mathrm{~km}$ (and approximately $2.5 \mathrm{~h}$ drive) from the capital city. The hospital was built as a joint venture between $\mathrm{RMOH}, \mathrm{PIH}$, and Clinton Health Access Initiative. Upon its inauguration in January 2011, the hospital had 152 beds and departments in emergency, general medicine, pediatrics, surgery, maternity, two operating theatres and a neonatal intensive care unit. As of June 2012, the hospital had 160 employees (including 67 medical and 30 paramedical). The state-of-the-art design and record for outstanding health achievements in Burera District [12] made Butaro hospital suitable for a model oncology program. A 27-bed cancer ward was converted for inpatient care, and a weekly cancer outpatient clinic was integrated into the non-communicable diseases clinic roster.

\section{Personnel and training}

All doctors and nurses at BCCOE received foundational didactic training through the national baseline cancer training, a 5-day didactic program - developed by partners including $\mathrm{RMOH}, \mathrm{PIH}, \mathrm{DFBWCC}$ - that covers general principles in cancer epidemiology, diagnosis, treatment, and documentation. Selected nurses additionally underwent an 8-week practicum-based longitudinal chemotherapy mixing and administration course led by visiting DFBWCC oncology specialty nurses. These trainings have facilitated long-term capacity building so that as of December 2014, 270 clinicians have received 
national baseline cancer training, and 36 nurses received the longitudinal training. Furthermore, three BCCOE Rwandan nurses have been recognized as national expert trainers, one of whom co-leads BCCOE-based longitudinal training offered to nurses from the National Referral Hospital of Kigali (CHUK).

\section{Clinical services}

Upon its opening, BCCOE provided histopathology-based diagnosis [13], X-ray and ultrasound imaging, chemotherapy, selected surgical procedures, palliative care and socioeconomic supports [14] delivered by a multidisciplinary team (Table 1). Patients requiring radiotherapy were referred to Mulago Hospital in Uganda. With no oncology specialists on-site, care was delivered through task-shifting and structured twinning, and long-term collaboration between BCCOE and DFBWCC [7, 8]. Generalist physicians prescribed chemotherapy and performed biopsies (including breast core-needle and bone marrow) while nurses mixed and administered chemotherapy. Clinicians followed standardized protocols and consulted teams of DFBWCC-based experts through weekly 'tumor boardlike' conference calls and frequent emails.

\section{Treatment protocols}

Care was standardized using protocols adapted to available resources and for non-oncologist clinicians [7, 8]. Led by $\mathrm{RMOH}$ and supported by $\mathrm{BCCOE}$ staff, protocols were drafted by international oncology experts and reviewed by the national Non-communicable Diseases (NCD) technical working group on an on-going basis. Given Rwanda's current lack of a radiotherapy center, treatment maximized outcomes without radiotherapy. The methodology for development and the initial vetting of protocols occurred at a conference held in Kigali, attended by cancer experts from France, USA, South Africa, and Senegal. The first national cancer protocols were endorsed in June 2012 (Table 2).

\section{Socioeconomic supports and access}

Complementing the medical services available, socioeconomic supports such as food packages and transport vouchers were critical for vulnerable patients (who were identified using standardized socioeconomic and clinical criteria). Additionally, given their prohibitive costs for the vast majority of patients, chemotherapy, pathology testing, and referral to Uganda for radiotherapy were free for all presenting patients and covered by funding from grants, foundations, and private donations. For all other hospital-related costs, most patients paid $10 \%$, with the remainder covered by the national communitybased health insurance scheme, Mutuelles de Sante (Mutuelles).

Table 1 Staffing at BCCOE

\begin{tabular}{|c|c|c|c|c|c|}
\hline & & Initial projections ${ }^{a}$ & Dec 2012 & Dec 2013 & Dec 2014 \\
\hline \multirow[t]{13}{*}{ Clinical } & Physicians & & & & \\
\hline & Internists & 1 & 1 & 2 & 2 \\
\hline & Pediatricians & 1 & 1 & 1 & 1 \\
\hline & General Practitioners & 1 & 1 & 2 & 2 \\
\hline & General Surgeon $^{\mathrm{b}}$ & 0.5 & 0.5 & 0.5 & 0.5 \\
\hline & OB/GYN & 0.5 & 0.5 & & 0.5 \\
\hline & Nurses & & & & \\
\hline & Inpatient & 7 & 7 & 13 & 22 \\
\hline & Outpatient $^{c}$ & 2 & 2 & 2 & 3 \\
\hline & Care Coordinator & 1 & 1 & 1 & 1 \\
\hline & Histopathology Technicians & - & 2 & 2 & 4 \\
\hline & Nutritionist & - & 0 & 0 & 1 \\
\hline & Social Worker & - & 0 & 0 & 1 \\
\hline \multirow[t]{5}{*}{ Programmatic } & Program Director & 1 & 1 & 1 & 1 \\
\hline & Program Manager & 1 & 1 & 1 & 1 \\
\hline & Administrative Assistant & - & 0 & 1 & 1 \\
\hline & Research Assistant & - & 0 & 1 & 1 \\
\hline & Data Officer & - & 0 & 1 & 1 \\
\hline
\end{tabular}

\footnotetext{
${ }^{\mathrm{a}}$ Initial projections based upon $\mathrm{MOH}$ estimates for 27-bed unit and $25 \%$ annual increase in patient population
}

${ }^{b}$ provide care for cancer and non-cancer patients

'Outpatient nurses shared with the NCD clinic 
Table 2 Outline of Rwanda national cancer protocols, using breast cancer as an example

\section{Each protocol: \\ - Places evidence-based practices in the context of national resources. Where clinical trials specific to resource-constrained settings have been conducted, associated protocols are applied (e.g. nephroblastoma, acute lymphoblastic leukemia, and Burkitt lymphoma). \\ - Is organized in a consistent format, with each protocol including subsections on screening, presenting signs and symptoms, pathology- based diagnosis, staging, treatment, and long-term follow up. \\ - Specifies the minimal essential work-up required to yield accurate, pathology-based diagnosis and inform management decision- making within the treatment options available. At BCCOE, testing for HER2 status is not routinely performed given limited availability of HER2-targeted therapies such as trastuzumab. \\ - Reflects staging classification that is clinically relevant and in line with treatment options. Three broad classifications/treatment groups for breast cancer are: early, locally advanced and metastatic. \\ - Takes into account the currently limited availability of radiotherapy. Mastectomy (with level I/II lymph node dissection) is prioritized as surgical treatment of choice over lumpectomy. \\ - Allows flexibility to address socioeconomic and logistical challenges seen in these settings. Weekly dosing of paclitaxel is employed where possible, however every three weeks dosing is offered given fewer barriers associated with the fewer hospital visits.}

For more detailed reference, copies of individual protocols are available upon request.

\section{Procurement}

A formulary list was generated from standardized protocols. Most medications were off-patent and included in the World Health Organization's essential medicines list. This list also included supplies such as infusion pumps and personal protective equipment. Procurement planning transitioned from ad hoc purchases before 2012 for the few cancer patients to stock orders made every 6-12 months by 2014. During the first year, consumption was tracked intensively with monthly manual stock counts and projections based on patient volume. These consumption data were reviewed quarterly, and orders made for anticipated stock outs within 6 months. Available drugs and consumables were procured through the public supply chain while $\mathrm{PIH}$ obtained the remainder using funding and DFBWCC donations. The above was performed by a PIH-employed pharmacist, working closely with and capacitating Butaro Hospital pharmacist, pharmacy technicians and relevant clinical program managers.

\section{Electronic Medical Records (EMR) system}

An oncology-specific EMR system was built on an opensource OpenMRS platform, borrowing principles from HIV medical record systems $[15,16]$. The database was devised to run off local servers, enabling work during internet interruptions. With the exception of chemotherapy ordering performed by clinicians, data entry of demographic data and clinical events was conducted by a dedicated data officer who had 2 years post-secondary school training. A team of a systems analyst, software developers, data officer, program managers, and clinicians developed and implemented this oncology-focused EMR system.

\section{Ethics}

Data related to human subjects presented in this manuscript is covered under a study protocol approved by Institutional Review Boards in Rwanda (National Health Research Council and Rwanda National Ethics Committee) and USA (Partners Human Research Committee). Given the retrospective design of this study and the use of de-identified data for analysis, informed consent was not required by respective Institutional Review Boards.

\section{Results: early findings, challenges faced and lessons learned Impact}

Between July 1, 2012 and June 30, 2014, 2326 patients presented to BCCOE for cancer-related evaluation or care. This is in contrast to 21 patients seen at Butaro hospital for cancer-related evaluation and care in the preceding 12 month period. Of these 2326 patients, 1640 (70.5\%) were female. Mean age was 43 years (standard deviation, SD, 19.8) and $270(11.6 \%)$ were children younger than 18 years of age (Table 3 ).

The total number of yearly outpatient visits at Butaro hospital increased from 17,895 in 2011 to 20,235 in the program's first year. During this period, the proportion of cancer-related outpatient visits also rose from 0.5 to $16 \%$. The increase in cancer-related hospital admissions was even more pronounced with $41 \%$ of 6583 admissions between July 1, 2013 and June 30, 2014 being cancer-related (Butaro Hospital Health Management Information System data, unpublished).

Of the 1144 patients who presented during BCCOE's first year (July 1, 2012 to June 30, 2013), 759 (66.3 \%) were diagnosed with cancer (Table 3). Of these, 519 $(68.4 \%)$ were female and $102(13.3 \%)$ children. Fiftyseven $(7.5 \%)$ were HIV-positive by self-report and 150 (19.8\%) had a smoking history. A high proportion (461, $60.7 \%$ ) presented with good functional status ECOG of $\leq 2$ [17]. Five hundred and sixty-four patients $(74.3 \%$ of cancer patients, or $98.4 \%$ of those with documented insurance status) were on Mutuelles. Sixty-seven patients $(8.8 \%)$ resided in Burera District and 11 (1.5\%) in neighboring countries. Five hundred and forty-nine (72.3 \%) were referred from district and national referral hospitals.

Pathology documentation was available for 562 patients (49.1\% of all patients presenting during BCCOE's first year, or $74.0 \%$ of patients diagnosed with cancer). As of June 30 , 
Table 3 Demographic and clinical characteristics of patients seen at BCCOE during first year

\begin{tabular}{|c|c|c|c|c|}
\hline \multirow[t]{2}{*}{ Characteristics } & \multicolumn{2}{|c|}{$\begin{array}{l}\text { Patients presenting for cancer evaluation/care in } \\
\text { year one }(n=1144)\end{array}$} & \multicolumn{2}{|c|}{$\begin{array}{l}\text { Patients diagnosed with cancer } \\
\text { in year one }(n=759)\end{array}$} \\
\hline & $\bar{n}$ & $\%$ & $\bar{n}$ & $\%$ \\
\hline Age & 1144 & 100.00 & 759 & 100.00 \\
\hline$<18$ & 135 & 11.80 & 102 & 13.44 \\
\hline $18-45$ & 470 & 41.08 & 247 & 32.54 \\
\hline $46-60$ & 334 & 29.20 & 256 & 33.73 \\
\hline$>60$ & 205 & 17.92 & 154 & 20.29 \\
\hline Gender & 1144 & 100.00 & 759 & 100.00 \\
\hline Male & 355 & 31.03 & 240 & 31.62 \\
\hline Female & 789 & 68.97 & 519 & 68.38 \\
\hline Residence Province & 1144 & 100.01 & 759 & 100.00 \\
\hline Northern Province - within Burera District & 168 & 14.69 & 67 & 8.83 \\
\hline Northern Province- OUTSIDE Burera District & 226 & 19.76 & 136 & 17.92 \\
\hline Other Provinces within Rwanda & 702 & 61.36 & 522 & 68.77 \\
\hline Outside Rwanda & 13 & 1.14 & 11 & 1.45 \\
\hline Unknown or not documented & 35 & 3.06 & 23 & 3.03 \\
\hline Type of referring facility & 1144 & 100.00 & 759 & 100.00 \\
\hline Referral or District Hospital & 735 & 64.25 & 549 & 72.33 \\
\hline Other type of facility (including outside Rwanda) & 153 & 13.37 & 90 & 11.86 \\
\hline Not documented & 256 & 22.38 & 120 & 15.81 \\
\hline Insurance Status & 1144 & 100.00 & 750 & 98.82 \\
\hline Mutuelles & 821 & 71.77 & 564 & 74.31 \\
\hline Other or not documented & 323 & 28.23 & 186 & 24.51 \\
\hline HIV status at intake & 1144 & 100.00 & 759 & 100.00 \\
\hline Positive & 67 & 5.86 & 57 & 7.51 \\
\hline Negative & 500 & 43.71 & 367 & 48.35 \\
\hline Unknown or not documented & 577 & 50.43 & 335 & 44.14 \\
\hline Smoking history & 1144 & 100.00 & 759 & 100.01 \\
\hline Current of previous & 189 & 16.52 & 150 & 19.77 \\
\hline Never & 645 & 56.38 & 471 & 62.06 \\
\hline Unknown or not documented & 310 & 27.10 & 138 & 18.18 \\
\hline Performance status at intake (ECOG) ${ }^{a}$ & & & 759 & 100.00 \\
\hline 0 & & & 313 & 41.24 \\
\hline 1 & & & 102 & 13.44 \\
\hline 2 & & & 46 & 6.06 \\
\hline 3 & & & 29 & 3.82 \\
\hline 4 & & & 13 & 1.71 \\
\hline Unknown or not documented & & & 256 & 33.73 \\
\hline
\end{tabular}

${ }^{\mathrm{a} E C O G: ~ E a s t e r n ~ C o o p e r a t i o n ~ O n c o l o g y ~ G r o u p ~[17] ~}$

2013, 95 (12.5 \%) cancer patients had died. Cause of death was documented as cancer-related for 24 (25.6\%), and unknown for 66 (69.5\%). Thirty-six (37.9\%) patients died at home or in the community while $45(47.4 \%)$ died during admission at BCCOE or another facility.
Discussion

Patients served

Butaro Cancer Center of Excellence has begun to deliver cancer services to a large number of patients in need in Rwanda (Table 3). Patients come from across the country, 
most residing in rural districts and covered by Mutuelles, thus indicating delivery to our target vulnerable populations. The unprecedented patient volume reflects the great need and highlights BCCOE's service as a national referral hospital for cancer care.

\section{Cancers seen}

Among adults, the most common diagnoses were breast cancer (189, $28.8 \%$ ), cervical cancer (141, $21.5 \%)$, and non-Kaposi sarcoma skin cancer (46, 7.0 \%). Among children, nephroblastoma (28, $27.5 \%$ ), acute lymphoblastic leukemia/ALL (25, $24.5 \%$ ), and Hodgkin lymphoma (10, $9.8 \%$ ) were the leading diagnoses (Table 4). Cancers seen at BCCOE reflect some of the regional trends, such as the two most common cancers being breast and cervical. In its first year, BCCOE would have seen half of all breast cancer cases expected to be diagnosed nationally based on
GLOBOCAN's estimates of 576 new breast cancer diagnoses annually in Rwanda [1], though the true national incidence and prevalence is currently unknown given robust registries to more accurately document cancer cases continue to be under development. The leading pediatric cancer at $\mathrm{BCCOE}$ was nephroblastoma. At $27 \%$ of pediatric cancers, this proportion was comparable to sites in the region such as in Zambia [18], though significantly higher than $5 \%$ among pediatric cancers in the United States [19]. The second most prominent pediatric cancer, ALL, was similarly common internationally $[1,19]$.

The distribution of cancers seen at BCCOE was influenced by variation in clinical resources across facilities in Rwanda, as well as patient selection. Prostate and gastric cancers, among the top five cancers in the region [1] were anecdotally less commonly seen at BCCOE than the

Table 4 Types of cancers diagnosed in patients enrolled at BCCOE during first year

\begin{tabular}{|c|c|c|c|c|c|}
\hline \multicolumn{6}{|l|}{ Cancers only $(n=759)$} \\
\hline & $n$ & $\%$ & & $\mathrm{n}$ & $\%$ \\
\hline Cancer Type Adults (18 years or older) & 657 & 100.0 & Cancer Type Children (<18 years) & 102 & 100.0 \\
\hline Breast cancer & 189 & 28.8 & Nephroblastoma & 28 & 27.5 \\
\hline Cervical Cancer & 141 & 21.5 & $\mathrm{ALL}^{\mathrm{a}}$ & 25 & 24.5 \\
\hline Non-Kaposi skin cancer & 46 & 7.0 & Hodgkin lymphoma & 10 & 9.8 \\
\hline Head and neck cancer & 38 & 5.8 & Rhabdomyosarcoma & 7 & 6.9 \\
\hline Colorectal cancer & 26 & 4.0 & Burkitt Lymphoma & 5 & 4.9 \\
\hline Other Gynecological Malignancies & 26 & 4.0 & Osteosarcoma & 5 & 4.9 \\
\hline $\mathrm{CML}^{\mathrm{a}}$ & 22 & 3.4 & Head and neck cancer & 4 & 3.9 \\
\hline Other Non Hodgkin's lymphoma & 21 & 3.2 & Other Leukemias & 4 & 3.9 \\
\hline Gastric cancer & 21 & 3.2 & Other Non Hodgkin's lymphoma & 3 & 2.9 \\
\hline Kaposi sarcoma & 16 & 2.4 & Other Bone Cancers & 3 & 2.9 \\
\hline Prostate cancer & 16 & 2.4 & Kaposi's sarcoma & 2 & 2.0 \\
\hline Other $\mathrm{Gl}^{\mathrm{a}}$ & 14 & 2.1 & Other Gynecological Malignancies & 2 & 2.0 \\
\hline Hodgkin's lymphoma & 13 & 2.0 & Breast cancer & 1 & 1.0 \\
\hline Soft-tissue Sarcomas & 12 & 1.8 & $C M L^{a}$ & 1 & 1.0 \\
\hline Other GU & 11 & 1.7 & Metastatic (unknown primary) & 1 & 1.0 \\
\hline Other solid cancers & 9 & 1.4 & Skin cancer & 1 & 1.0 \\
\hline Other Leukemias & 8 & 1.2 & & & \\
\hline$A L L^{a}$ & 7 & 1.1 & & & \\
\hline Metastatic (unknown primary) & 5 & 0.8 & & & \\
\hline Multiple myeloma & 5 & 0.8 & & & \\
\hline Osteosarcoma & 3 & 0.5 & & & \\
\hline Unknown type & 2 & 0.3 & & & \\
\hline Rhabdomyosarcoma & 2 & 0.3 & & & \\
\hline Other Bone Cancers & 2 & 0.3 & & & \\
\hline Large B-cell lymphoma & 1 & 0.2 & & & \\
\hline Nephroblastoma & 1 & 0.2 & & & \\
\hline
\end{tabular}

${ }^{a} C M L$ chronic myeloid leukemia, Other Gl other gastrointestinal cancers, Other GU other genitourinary cancers, ALL acute lymphoblastic leukemia 
national referral hospital CHUK, which has resident endoscopists and urologists. Strengthening of the national cancer registry will provide a more accurate epidemiologic picture of cancers in Rwanda, though diagnostic capacity is limited in much of the country so many patients remain undiagnosed and therefore uncounted. In addition to teasing out the role of diagnostic and referral bias, further studies may be needed to explore region-specific risk factors for cancers.

\section{Outcomes}

It is too early to describe disease-specific clinical outcomes, planned for the near future. Of note, however, of the 95 documented deaths, that the majority (53, $55.8 \%$ ) of patients die at home or while admitted at another facility makes discerning cause of death difficult and partly explains the large number $(66,69.5 \%)$ of deaths with unknown cause.

\section{Challenges and lessons learned Documentation gaps}

The current oncology EMR needs further development but serves as a starting point. Many EMR systems for HIV in resource-constrained settings have demonstrated positive impact $[15,16]$, however few if any published examples describe systems for cancer care. While data gaps have to be addressed and clinical impact of BCCOE's EMR system to be assessed, we have used this system to generate the presented data and routinely to support clinical management and program development. EMR data has, for instance, improved tracking of patients who miss appointments and prioritization of protocol revisions. While the initial phase of EMR development emphasized data supporting management decisions and patient retention, the next phase aims to better capture disease-specific outcomes, evaluate protocol adherence, and monitor treatment toxicity.

\section{Staff shortages}

Unprecedented patient volume contributed to perpetual staffing shortages, requiring periodic review. The initial seven ward nurses increased to 26 as of August 2014, corresponding to a nurse: inpatient ratio of 1:8. Despite the higher patient volume, in-service training, and heavy emotional toll of their work, oncology nurses are currently paid the same as those in other departments. Temporary relief has been achieved by increasing staff using personnel allocated by $\mathrm{RMOH}$ and additional funding from partners. However, strategies for compensation and professional accreditation for nurses and doctors will be necessary to sustain a cadre of oncology-skilled clinicians [8].

Butaro Cancer Center of Excellence does not have an onsite gynecologist or pathologist, and has only intermittently had a surgeon $[13,14]$. While recruitment is in process and longer-term local capacity is developed through in-country post-graduate programs [8], discussions are underway for BCCOE to serve as a national oncology rotation site for post-graduate doctors. The stream of students and affiliated rotating faculty may mitigate staffing shortages while presenting valuable opportunities for learning and collaboration.

\section{Access to radiotherapy}

Over $50 \%$ of cancer cases in LMICs countries are estimated to require radiotherapy [20], yet Rwanda does not have a radiotherapy facility. PIH sponsored an average of 15 patients a month to receive radiotherapy at Uganda's Mulago Hospital. Due to budget constraints, a committee of clinicians used institutional guidelines to select a subset of eligible patients, mainly with curable cervical and head and neck cancers. The average cost per patient receiving a 6-week course of chemo-radiation for locally advanced cervical cancer (includes transport, room and board, and medical services) was USD 2800, amounting to over USD 500,000 spent per year. Systematic study of patient outcomes following referral for radiation at Mulago is planned. Discussions are ongoing to build a radiotherapy facility within the next 5 years- a criticallyneeded investment to expand treatment options for Rwandans.

\section{Program expenses}

The most significant costs in BCCOE's program budget were radiotherapy referrals, chemotherapy, and staff salaries. Chemotherapy orders during BCCOE's first year amounted to USD 110,000 while clinical staff salaries totaled USD 312,000. Though a substantial amount, funding can be within reach and costs significantly reduced through partnerships. As examples, salaries for USlicensed specialists supporting cancer care were subsidized through part-time hospitalist work in the US. Medications were sourced from accredited India-based generic drug companies whose prices were three to five times cheaper than European counterparts [21]. In addition to these program-level strategies, it is our hope that through advocacy and price negotiation, global financing mechanisms for cancer care will become a reality, as was accomplished for antiretroviral therapy.

\section{Sustainability}

The scale of BCCOE's work and its funding can be attributed to the broad and invested collaborations supporting it. While we currently have not yet enumerated the cost of implementing BCCOE (an exercise that is currently in process), approaches have been made to minimize costs and facilitate sustainability of the program. RMOH's engagement and role in financing and shepherding partnerships has not only helped frame national priority, but has 
provided an avenue for continuation of services over the long-term and their integration into the existing health infrastructure. Use of open-source data systems and of rotating volunteer experts have also minimized costs. On a global scale partners are participating in efforts with World Health Organization and manufacturing companies geared toward lowering prices of chemotherapeutic agents and improving access internationally.

\section{Conclusion}

Butaro Cancer Center of Excellence demonstrates that, with partnerships and supports, complex cancer care can be delivered in the most resource-constrained settings and despite significant challenges. Key attributes that have made BCCOE possible were: a) meaningful partnerships emphasizing health systems strengthening, b) innovative task- and infrastructure-shifting, c) strong $\mathrm{RMOH}$ leadership coordinating efforts to embed services within the public sector, and d) an equity-driven agenda to service those most in need. This combination is rare yet essential to expand desperately needed cancer services globally. Looking forward, clinical outcomes will be assessed such as long-term survival, retention and treatment-related toxicity for specific cancers treated. The developed EMR system will serve as a valuable platform for this assessment. Finally, these partnerships continue to grow and support national efforts, including periodic review of national protocols to reflect experience since 2012 and planning for an in-country radiotherapy center.

\section{Availability of data and materials}

Presented data are available upon request from corresponding author.

\begin{abstract}
Abbreviations
LMICs: low- and middle-income countries; RMOH: Rwanda Ministry of Health; $\mathrm{PIH}$ : partners in health; DFBWCC: Dana-Farber/Brigham and Women's Cancer Center; BCCOE: Butaro Cancer Center of Excellence; EMR: electronic medical records; NCD: non-communicable diseases; SD: standard deviation;

ALL: acute lymphoblastic leukemia.

\section{Competing interests}

The authors (NT, TM, BH-G, MM, EM, MJX, IN, VH, DGU, LEP, JBB, JJW, CD, $F R U, P C D, G N, C S, C M, L L$ and $L N S$ ) declared no conflict of interest and none have been paid to write this article by a pharmaceutical company or other agency. This study was not funded by any outside organization. The funders for BCCOE program had no role in study design, data collection, data analysis, data interpretation or writing of this manuscript. The corresponding author had full access to all the data in this study and had final responsibility for the decision to submit for publication. The authors confirm that this study has not been submitted to any other medical journal, and has not been previously published in any medical journal.
\end{abstract}

\section{Authors' contributions}

NT conducted literature review, assisted with organization of manuscript, supported data collection and cleaning, performed data analysis and interpretation, and wrote and edited the manuscript. LNS assisted with the organization of the manuscript, interpretation of the data, reviewed and edited the manuscript. TM and LL assisted with organization of the manuscript, interpretation of the data, and reviewed and edited manuscript. $\mathrm{BH}-\mathrm{G}$ performed data analysis and interpretation, assisted with organization of the manuscript, and reviewed and edited manuscript. MJX helped with literature review, design of tables, and reviewed and edited the manuscript. $\mathrm{CD}$ and FRU collected and cleaned data, and reviewed and edited the manuscript. MM, EM, IN, VH, DGU, LEP, JBB, JJW, PCD, GN, CS and CM helped to review and edit the manuscript. All authors read and approved the final manuscript.

\section{Acknowledgments}

The authors would like to thank the oncology clinical and EMR teams at Butaro Cancer Center of Excellence for their dedicated work, the Dana Farber/Brigham and Women's Cancer Center, Brigham and Women's Hospital Division of Global Health Equity, Boston Children's Hospital, Harvard Medical School Department of Global Health and Social Medicine Research Core, Non-Communicable Diseases Writing Group, Rwanda Ministry of Health leadership, National University of Rwanda for scholarship and mentorship, Lori Buswell, Temidayo Fadelu, Fidel Rubagumya, Cheryl Amoroso, Sara Stulac, Gaspard Muvugabigwi, Gene Bukhman, Irenee Nshimiyimana, Shekinah Elmore and Aimee Muhimpundu. We are also grateful to Jeff Gordon Children's Foundation, Livestrong Foundation, GlaxoSmithKline, Breast Cancer Research Foundation, Max Foundation, Dana Farber/Brigham and Women's Cancer Center, Partners In Health, Rwanda Ministry of Health and Rwanda Biomedical Center for funding and other supports for patient care that have made this work possible.

\section{Author details}

${ }^{1}$ Botswana Ministry of Health, Gaborone, Botswana. ²Partners In Health/ Inshuti Mu Buzima, Kigali, Rwanda. ${ }^{3}$ Dana-Farber/Brigham \& Women's Cancer Center, Boston, USA. ${ }^{4}$ Harvard Medical School, Boston, USA. ${ }^{5}$ Rwanda Ministry of Health, Kigali, Rwanda. ${ }^{6}$ Boston Children's Hospital, Boston, USA. ${ }^{7}$ University of Vermont College of Medicine, Burlington, USA. ${ }^{8}$ Icahn School of Medicine at Mount Sinai, New York, USA. ${ }^{9}$ Abramson Cancer Center, University of Pennsylvania, Philadelphia, USA. ${ }^{10}$ Division of Global Health Equity, Brigham and Women's Hospital, Boston, USA.

Received: 25 October 2015 Accepted: 8 March 2016

Published online: 18 March 2016

References

1. Ferlay J, Soerjomataram I, Ervik M, Dikshit R, Eser S, Mathers C, et al. GLOBOCAN 2012 v1.0, Cancer incidence and mortality worldwide: IARC CancerBase No. 11 [Internet]. Lyon: International Agency for Research on Cancer; 2013. Available: http://globocan.iarc.fr. Accessed 15 July 2014.

2. Farmer P, Frenk J, Knaul FM, Shulman LN, Alleyne G, Armstrong A, et al. Expansion of cancer care and control in countries of low and middle income: A call to action. Lancet. 2010;376(9747):1186-93.

3. Binagwaho A, Ngabo F, Wagner CM, Mugeni C, Gatera M, Nutt CT, et al. Integration of comprehensive women's health programmes into health systems: cervical cancer prevention, care and control in Rwanda. Bull World Health Organ. 2013;91:697-703.

4. Knaul FM, Farmer P. Overview. In: Knaul FM, Gralow JR, Atun R, Bhadelia A, editors. Closing the cancer Divide: an Equity Imperative. Cambridge: Harvard Global Equity Initiative/Harvard University Press; 2012. p. 3-28.

5. Stefan DC, Elzawawy AM, Khaled HM, Ntaganda F, Asiimwe A, Addai BW, et al. Developing cancer control plans in Africa: Examples from five countries. Lancet Oncol. 2013;14:e189-95.

6. Strother RM, Asirwa FC, Busakhala NB, Njiru E, Orang'o E, Njuguna F, et al. The evolution of comprehensive cancer care in Western Kenya. J Cancer Policy. 2013;1:e25-30.

7. Shulman LN, Mpunga T, Tapela N, Wagner CM, Fadelu T, Binagwaho A. Bringing cancer care to the poor: Experiences from Rwanda. Nat Rev Cancer. 2014;142(4):541-5.

8. Stulac S, Binagwaho A, Tapela NM, Wagner CM, Muhimpundu MA, Ngado F, et al. Capacity building for oncology programs in sub-Saharan Africa: the Rwanda experience. Lancet Oncol. 2015;16(8):e405-13.

9. Binagwaho A, Farmer PE, Nsanzimana S, Karema C, Gasana M, de Dieu Ngirabega J, et al. Rwanda 20 years on: investing in life. Lancet. 2014; 384(9940):371-5. 
10. Binagwaho A, Wagner CM, Gatera M, Karema C, Nutt CT, Ngabo F. Achieving high coverage in Rwanda's national human papillomavirus vaccination programme. Bull World Health Organ. 2012;90:623-8.

11. Rich ML, Miller AC, Niyigena P, Franke MF, Niyonzima JB, Socci A, et al. Excellent clinical outcomes and high retention in care among adults in a community-based HIV treatment program in rural Rwanda. J Acquir Immune Defic Syndr. 2012;59(3):e35-42.

12. Government of Rwanda, Northern Province Burera District: Burera District Health Strategic Plan 2012-2018. Republic of Rwanda. May 2013. Print.

13. Mpunga T, Tapela N, Hedt-Gauthier BL, Milner D, Nshimiyimana I, Muvugabigwi G, et al. Diagnosis of cancer in rural Rwanda: Early outcomes of a phased approach to implement anatomic pathology services in resource-limited settings. Am J Clin Pathol. 2014;142:541-5.

14. Mubiligi JM, Hedt-Gauthier B, Mpunga T, Tapela N, Okao P, Harries AD, et al. Caring for patients with surgically resectable cancers: experience from a specialized center in rural Rwanda. Public Health Action. 2014;4(2):128-32.

15. Tierney WM, Rotich JK, Hannan TJ, Siika AM, Biondich PG, Mamlin BW, et al. The AMPATH medical record system: creating, implementing, and sustaining an electronic medical record system to support HIV/AIDS care in western Kenya. Stud Health Technol Inform. 2007;129:372-6.

16. Amoroso CL, Akimana B, Wise B, Fraser HSF. Using electronic medical records for HIV care in rural Rwanda. Stud Health Technol Inform. 2010;160:337-41.

17. Oken MM, Creech RH, Tormey DC, Horton J, Davis TE, McFadden ET, et al. Toxicity and response criteria of the Eastern Cooperative Oncology Group. Am J Clin Oncol. 1982;5:649-55.

18. Slone JS, Chunda-Liyoka C, Perez M, Mutalima N, Newton R, Chintu C, et al. Pediatric malignancies, treatment outcomes and abandonment of pediatric cancer treatment in Zambia. PLoS One. 2014;9(2):e89102.

19. American Cancer Society. Cancer Facts \& Figures 2014. Atlanta: American Cancer Society; 2014.

20. Barton MB, Frommer M, Shafiq J. Role of radiotherapy in cancer control in low-income and middle-income countries. Lancet Oncol. 2006;7:584-95.

21. Lopes Gde L. Cost comparison and economic implications of commonly used originator and generic chemotherapy drugs in India. Ann Oncol. 2013;24 Suppl 5:v13-6.

\section{Submit your next manuscript to BioMed Central and we will help you at every step:}

- We accept pre-submission inquiries

- Our selector tool helps you to find the most relevant journal

- We provide round the clock customer support

- Convenient online submission

- Thorough peer review

- Inclusion in PubMed and all major indexing services

- Maximum visibility for your research

Submit your manuscript at www.biomedcentral.com/submit

) Biomed Central 